\title{
High-dose PPI-Amoxicillin Dual Therapy for First-Line Helicobacter pylori Therapy
}

\author{
Tran Thi Khanh Tuong* \\ Department of Internal Medicine, Pham Ngoc Thach University of Medicine, Vietnam
}

*Corresponding author: Tran Thi Khanh Tuong, Department of Internal Medicine, Pham Ngoc Thach University of Medicine, 02 Duong Quang Trung, District 10, Ho Chi Minh city, Vietnam

ARTICLE INFO

Received: 慧 April 03, 2020

Published: April 20, 2020

Citation: Tran Thi Khanh Tuong. High-dose PPI-Amoxicillin Dual Therapy for First-Line Helicobacter pylori Therapy. Biomed J Sci \& Tech Res 27(1)-2020. BJSTR. MS.ID.004452.

Keywords: High Dose Dual Therapy; Eradication; Helicobacter pylori infection

\section{ABSTRACT}

Background: The efficacy of treatment of Helicobacter pylori (H. pylori) infection has decreased because of increasing resistance to clarithromycin, metronidazole, and levofloxacin. Resistance to amoxicillin is generally low, and high intragastric $\mathrm{pH}$ increases the efficacy of amoxicillin, so we performed this study to assess the efficacy of a high-dose dual therapy in treatment-naive patients with $H$. pylori infection.

Methods: a total of 89 patients with $H$. pylori infection were recruited in University Medical Center Ho Chi Minh city. All patients underwent endoscopy before treatment. Four to eight weeks after completing the course of therapy, H. pylori infection status was examined by $\mathrm{C}^{13}$-urea breath tests. Patients were received a high-dose dual therapy (rabeprazole $20 \mathrm{mg}$ QID and amoxicillin $1000 \mathrm{mg}$ QID for 14 days).

Results: High-dose dual therapy achieved high efficacy of intention-to-treat (ITT) eradication rate, $84.3 \%$ (95\% CI $75.6-91.5 \%)$, and of per-protocol (PP) eradication rate $88.8 \%$ (95\% CI 79.5 - 93.9\%). The adverse event rates were $29.2 \%$. Compliance rate was $96.6 \%$.

Conclusion: A 14day rabeprazole- and amoxicillin-containing high-dose dual therapy achieves a high eradication rate as first-line anti-H. pylori therapy.

\section{Introduction}

The first 3-drug regimen for Helicobacter pylori infection $(H$. pylori) including Proton Pump Inhibitor (PPI) / clarithromycin / amoxicillin or metronidazole for 7-14 days is not currently recommended in areas with resistance. clarithromycin $>15 \%$, due to $H$. pylori eradication rates drop to unacceptable levels $(\leq 80 \%)$ $[1,2]$. The main reasons for failure of $H$. pylori infection include antibiotic resistance, poor adherence and rapid metabolism of Proton Pump Inhibitors (PPIs). Clarithromycin resistance is a major cause of failure of the standard triple regimen. So far, amoxicillinresistant $\mathrm{H}$ pylori is still rare in the world. Therefore, high-dose dual therapy amoxicillin-PPI is one of the Maastrich V (2017) consensus regimens recommended in the treatment of H. H. Pylori infection for the first time [2]. The three most important factors associated with the success of high-dose dual therapy include the ability to maintain stomach $\mathrm{pH} \geq 6$, the dose of amoxicillin and the interval between doses and duration of treatment. Recently, the study of Yang et al. Showed that a 14-day high-dose dual therapy consisting of $20 \mathrm{mg}$ rabeprazole and $750 \mathrm{mg}$ amoxicillin every 6 hours achieved an initial eradication rate of $H$. pylori of $95.3 \%$ [3]. However, another study from China when optimizing high dose amoxicillin and proton pump inhibitors did not achieve high H.pylori eradication rates [4]. The effectiveness of high-dose dual regimens in the treatment of $H$. pylori infection for the first time has not been reported in our country, so we implement this topic with the goal:

(1) Determination of $H$. Pylori eradication rate of high-dose dual regimen (rabeprazole - amoxicillin) in patients with gastric or duodenal / gastric ulcer.

(2) Determine patient compliance and side effects for highdose dual regimens. 


\section{Materials and Methods}

Patients diagnosed with H. pylori infection who were indicated for treatment at the gastroenterology clinic of Ho Chi Minh City University of Medicine and Pharmacy

Inclusion Criteria: Patients 18 years of age and older diagnosed with $\mathrm{H}$. pylori infection who were indicated for treatment at the gastroenterology clinic of Ho Chi Minh City University of Medicine and Pharmacy.

Patients diagnosed with $H$. pylori infection when positive urease test (CLO test) was indicated for H.pylori treatment including

\section{Exclusion Criteria:}

1. Stomach / duodenal ulcer diagnosed by upper gastrointestinal endoscopy

2. Functional dyspepsia according to ROME IV-2016 standards Exclusion criteria

3. Stomach cancer (diagnosed with endoscopy with or without pathology)

4. Severe medical conditions: decompensated cirrhosis, end-stage chronic kidney disease, severe heart failure, COPD...

5. Patient is using antibiotics other than those mentioned in the above regimen

6. The patient has no prescription other than a prescription for H. pylori infection for a period of 14 days

7. Pregnant or lactating women

All patients will have upper endoscopy and CLO test. Patients who were eligible for the study will be treated with high-dose double therapy for 14 days, without any combination of any other digestive drugs besides 2 drugs in the regimen such as probiotics, prokinetic drugs), antacid... with time, dosage as follows:

1. Rabeprazole 20mg (Pariet) 1 capsule $\mathrm{x} 4$ times daily for 5.5 hours, 30 minutes to 60 minutes before meals.

2. Amoxicillin 500mg 2 tablets 4 times / day, every 5.5 hours after a meal.

During treatment, all patients must eat 4 meals: breakfast, lunch, dinner and add a 4th appointment in the evening, the meals must have protein to stimulate gastric acid secretion. The patients were consulted about the effectiveness, how to take the medicine and the common side effects of the medicine. All prescriptions are carefully documented for how to take, possible side effects and re-examination dates, so that patients are not mistaken and less worried when experiencing side effects. Patients are given the phone number of the treating doctor to contact when needed. Patients were re-examined after 2-3 weeks to evaluate side effects and adherence. Check H.pylori status after 4-8 weeks of treatment with rapid urease test (CLO test) or C13 breath test (C13 ureabreath test). The patient did not take any other antibiotic or bismuth for at least 4 weeks, proton pump inhibitor for at least 2 weeks and $\mathrm{H} 2$ receptor antagonist for at least 1 week before rechecking for H.pylori infection. Evaluation of adherence: $<50 \%$ of oral medication is not compliant, $50-<80 \%$ is poorly adhered, $\geq 80 \%$ is adherent. Patients who are $\geq 80 \%$ compliant will be placed in the analysis group according to the study design (PP) All data were analyzed using software of Stata $12, \mathrm{p}<0.05$ is statistically significant. Use the $\chi 2$ test or calibrate Yate's to compare 2 ratios. Compare 2 averages by t tests.

\section{Results}

Most patients have endoscopy as gastritis, 9\% normal endoscopy results diagnosed as functional indigestion (Table 1). There were 4 patients who adhered to the treatment $<80 \%, 6$ patients did not re-examine after the end of treatment 4-8 weeks, so the number of patients analyzed according to the study design was 82 . High-dose dual therapy is highly effective in eradicating $H$. pylori (Table 2). The majority of patients had no side effects, the total number of patients with side effects was $28.6 \%$ and all side effects were mild (Table 3). Most of patients complied with $\geq 80 \%$ accounting for $94.5 \%$ (Table 4 ).

Table 1: Population characteristics.

\begin{tabular}{|c|c|}
\hline Characteristics & n (\%) \\
\hline Sex (male) & $42 / 91(47.2)$ \\
\hline Age & $42.5 \pm 11.9$ \\
\hline Gastric and duodenal ulcers & $13(14.3)$ \\
\hline Gastritis and duodenitis & $70(76.9)$ \\
\hline Normal & $8(98.8)$ \\
\hline $\begin{array}{c}\text { Comorbidities (Irritable bowel syndrome, } \\
\text { gastroesophageal reflux) }\end{array}$ & $28(31.5)$ \\
\hline Smoking habit & $12(13.5)$ \\
\hline Drinking habits & $17(19.1)$ \\
\hline
\end{tabular}

Table 2: H.pylori eradication rate

\begin{tabular}{|c|c|c|}
\hline & n & Rate $\%$ \\
\hline ITT & $73 / 91$ & 80.2 \\
$95 \%$ CI & $74 / 82$ & $75.6-90.2$ \\
\hline PP & 70.2 \\
$95 \%$ CI & & $79.5-95.9$ \\
\hline
\end{tabular}

Table 3: Side effects.

\begin{tabular}{|c|c|c|c|}
\hline Side effects & $\mathbf{n}$ & $\mathbf{\%}$ & Level \\
\hline Nausea & 3 & 3.3 & Light \\
\hline Diarrhea & 11 & 12.1 & Light \\
\hline Itching & 3 & 3.3 & Light \\
\hline $\begin{array}{c}\text { Boilling } \\
\text { abdomen }\end{array}$ & 17 & 18.7 & Light \\
\hline Total patients & 26 & 28.6 & \\
\hline
\end{tabular}


Table 4: Compliance level of treatment.

\begin{tabular}{|c|c|c|}
\hline Compliance level & n & \% \\
\hline$<50 \%$ & 0 & 0.0 \\
\hline $50-<80 \%$ & 5 & 5.5 \\
\hline$\geq 80 \%$ & 86 & 94.5 \\
\hline Total & 91 & 100 \\
\hline
\end{tabular}

\section{Discusion}

The high dose double dose system used in this study was based on two factors:

a) Low amoxicillin resistance worldwide. On the other hand, the bactericidal effect of amoxicillin on $H$. pylori is thought to be dependent on concentration over time (AUC), so higher doses several times a day will have better results.

b) $H$. pylori becomes non-replicating state and does not respond to antibiotics when stomach $\mathrm{pH}$ is $3-6$. When the stomach $\mathrm{pH}$ is increased to $6-8$, the bacteria will turn to a new multiplication state to respond to antibiotics [3]

Following the recommendations of the United States School of Gastroenterology (ACG 2017) [1], high-dose dual therapy consists of standard or double dose proton pump inhibitors administered three or four times daily in combination with $750 \mathrm{mg}$ or amoxicillin. $1000 \mathrm{mg}$ orally 3 or 4 times / day. We chose rabeprazole as PPI in our study because rabeprazole is not metabolized by the CYP2C19 pathway and is therefore not affected by polymorphism of this enzyme. In addition, rabeprazole is a 2nd generation PPI that inhibits strong acids. A stable level of acid suppression has been shown to be important in optimizing the effects of antibiotics such as amoxicillin, an acid-resistant antibiotic. Our study using $20 \mathrm{mg}$ of rabeprazole in combination with $1000 \mathrm{mg}$ of amoxicillin 4 times a day for 2 weeks showed that the eradication rates based on ITT and PP analysis were $80.2 \%$ and $90.2 \%$ lower than The study of Yang JC and colleagues in Taiwan on the group of patients treated with H. pylori infection for the first time reached 95.3\% [5]. However, the eradication rates in our door study were significantly higher than in a recent pooled study of $473 \mathrm{H}$.pylori-infected patients who failed their first eradication at $81.3 \%$ [6]. The elimination effect in the pooled study is not high, probably due to the heterogeneity between these studies on the type of PPI used and the dose of PPI, amoxicillin

Thus, the H.pylori eradication effect of this dual therapy is related to the type of PPI used, the dose of amoxicillin, and the number of daily doses. High-dose dual therapy has a frequency of using the drug 4 times a day at 5.5 hours, which is a difficult problem for patients. However, because all patients in our study were carefully consulted, the compliance rate was good, reaching $94.5 \%$. At the end of the study, 6 patients did not follow up again in a total of 91 patients enrolled in the original study. We assessed side effects by asking patients for side effects during treatment. These side effects were assessed on four levels: mild, moderate, severe and very severe. We noted that 26 patients $(28.6 \%)$ had side effects, but all these side effects were mild and transient, no patients reported severe side effects. The side effects of this therapy include: nausea, diarrhea, itching and intestinal boil, as noted by Kwack [7] and Yang JC [5]. The results of this study showed that the incidence of side effects of high-dose dual therapy was much lower than that of bismuth 4-drug regimen when the incidence of side effects of bismuth 4-drug regimen was recorded in the autistic children's study. The wall in 2017 [8] is $80.5 \%$. Other studies on high-dose dual therapy have shown that it is safe, well tolerated, and has side effects.

\section{Conclusion}

High-dose dual therapy containing rabeprazole and amoxicillin for 14 days achieved a high eradication rate in the treatment of $H$. pylori infection for the first time with a high compliance rate and few side effects.

\section{References}

1. Chey William D, Leontiadis Grigorios I, Howden Colin W, Steven F Moss (2017) ACG clinical guideline: treatment of Helicobacter pylori infection. The American journal of gastroenterology 112(2): 212-239.

2. Malfertheiner P, Megraud F, O Morain CA, JP Gisbert, EJ Kuiper, et al. (2017) Management of Helicobacter pylori infection-the Maastricht V/ Florence consensus report. Gut 66(1): 6-30.

3. Scott David R, Marcus Elizabeth A, Wen Yi, et al. (2007) Gene expression in vivo shows that Helicobacter pylori colonizes an acidic niche on the gastric surface. Proceedings of the National Academy of Sciences 104(17): 7235-7240.

4. Jia Li Hu, Jun Yang, Yin Bin Zhou, Jane Oh, George Sachs (2017) Optimized high-dose amoxicillin-proton-pump inhibitor dual therapies fail to achieve high cure rates in China. Saudi J Gastroenterol 23(5): 275-280.

5. Yang Jyh Chin, Lin Chun Jung, Wang Hong Long, Jin De Chen, John Y Kao, et al. (2015) High-dose dual therapy is superior to standard first-line or rescue therapy for Helicobacter pylori infection. Clinical Gastroenterology and Hepatology 13(5): 895-905.

6. Gao Cai Ping, Zhou Zhou, Wang Jia Zhen, Sheng Xi Han 1, Liang Ping Li, et al. (2016) Efficacy and safety of high-dose dual therapy for Helicobacter pylori rescue therapy: A systematic review and meta-analysis. Journal of digestive diseases 17(12): 811-819.

7. Kwack WonGun, Lim YunJeong, Lim ChiYeon, Graham David Y (2016) High Dose Ilaprazole/Amoxicillin as First-Line Regimen for Helicobacter pylori Infection in Korea. Gastroenterology research and practice: 1648047.

8. Tran Thi Khanh Tuong (2017) The efficacy of 4-medication protocol included Bismuth for in treatment Helicobacter Pylori infection. Vietnam Journal of Gastroenterology 49(9): 3067-3073. 
ISSN: 2574-1241

DOI: $10.26717 /$ BJSTR.2020.27.004452

Tran Thi Khanh Tuong. Biomed J Sci \& Tech Res

(C) This work is licensed under Creative

Submission Link: https://biomedres.us/submit-manuscript.php

$\begin{array}{ll}\text { BIOMEDICAL } & \text { Assets of Publishing with us } \\ \text { RESEARCHES } & \text { - Global archiving of articles } \\ & \text { - Immediate, unrestricted online access } \\ & \text { - Rigorous Peer Review Process } \\ & \end{array}$

